\title{
Automated abstraction of myocardial perfusion imaging reports using natural language processing
}

\author{
Parija Sharedalal, MD, ${ }^{\mathrm{a}}$ Ajay Singh, MBBS, MS, PhD, ${ }^{\mathrm{b}}$ Neal Shah, $M D,{ }^{\mathrm{c}}$ and \\ Diwakar Jain, MD, FACC, MASNCa \\ a Department of Cardiovascular Medicine, Westchester Medical Center, Valhalla, NY \\ b Senior Manager Artificial Intelligence, BioXcel Therapeutics Inc, New Haven, CT \\ c Department of Medicine, Westchester Medical Center, Valhalla, NY
}

Received Dec 3, 2020; accepted Dec 10, 2020

doi: $10.1007 / \mathrm{s} 12350-020-02507-4$

\section{See related article, pp. 1178-1187}

In today's world, artificial intelligence (AI) is intertwined with our everyday lives. Most people interact with AI every day whether it is facial recognition on smart phones, personalized feeds on social media, smart home devices, simple Google searches, or even buying something from Amazon. As such, the symbiosis of AI with the future of health care is inevitable. Over the last few years, the potential of AI and related applications (i.e., deep learning, machine learning, natural language processing) has exploded immensely. The potential role of AI in the healthcare industry, medical research, and clinical medicine is enormous.

AI has the potential to simplify the management and analysis of complex data, aiding physicians in their decision-making while improving clinical productivity. To explain this very complex field, AI can be understood as an attempt to replicate human intelligence and thought process via computer algorithms ${ }^{1}$. AI has the capability to recognize and highlight patterns, mine large data, and decipher meaningful relationships in a given data set. AI's use in clinical medicine is being explored in the fields of diagnosis, choosing optimal treatment plans, and for prediction of outcome. Two important sub-groups of AI applications, natural

Reprint requests: Diwakar Jain, MD, FACC, MASNC, Department of Cardiovascular Medicine, Westchester Medical Center, 100 Woods Road, Valhalla, NY 10595; dj2700@gmail.com

J Nucl Cardiol 2022;29:1188-90.

1071-3581/\$34.00

Copyright (C) 2021 American Society of Nuclear Cardiology. language processing (NLP) and machine learning, have proven to be valuable in healthcare and they allow for analysis of large amounts of unstructured data from the documentation that often makes up a large part of patient records.

The widespread use of electronic health records (EHRs) has amplified the amount of patient data accessible to physicians. The data, however, are often constrained by the design of a particular EHR and oftentimes include large volumes of unstructured data and free hand text. The challenging nature of complex, unstructured data has led to a disappointing experience amongst clinicians, particularly while trying to abstract and analyze accurate information from such data. This is further compounded by natural factors such as fatigue, distraction, and stress which interfere with the consistency and accuracy of human abstraction of data. Clinical natural language processing (cNLP) as described in this study by Zheng et al. ${ }^{2}$, in this issue of the journal, allows for abstraction and organizing of the data from free text. cNLP is trained to understand the unique medical language, abbreviations, and common medical jargon and output this text into structured information which renders itself for statistical analysis and perhaps can then be used to guide clinical decisions.

$\mathrm{AI}$ is promising in attaining precision. Fields such as radiology and pathology increasingly rely on AI to improve diagnostic accuracy. AI augments the proficiency of a radiologist or a histopathologist ${ }^{3}$ by reviewing the entire data to identify complex patterns in the entire data, quantify these characteristics and point these area of interest to the image reader ${ }^{4}$. This can help reduce cost by improving the efficiency of medical image readers. AI reduces redundancy and thereby may decrease physician workload and burnout. In this 
instance, while AI certainly works to improve efficacy, decrease physician fatigue, and streamline workflow, the notion that AI will replace healthcare workers in the future must be dismissed. AI's biggest limitation lies in that it only performs the task in which it has been trained. The most sophisticated AI algorithm is limited in that it is a single purpose algorithm. It can perform only one application-for which it has been trainedand perform it with extreme precision. A program designed to read one kind of radiological images e.g., thoracic CT can only examine thoracic CTs and cannot read any other organ system nor can it read images acquired with other imaging modalities e.g., thoracic MR or perfusion images. In contrast, human brain can absorb and process multiple problems, which may be unrelated to each other, at the same time and often seamlessly.

Wearable patient monitoring technologies such as smart watches are AI-enabled ${ }^{5}$. Wearable technology uses AI, including deep learning algorithms, for data processing. This is particularly helpful in the interpretation of cardiac data such as heart rate analysis and atrial fibrillation detection. Beyond consumer wearables, several medical wearables are also likely to get AI-enabled, with uses ranging from remote patient monitoring and health tracking to behavioral health. It is important to note that while many software companies have interest in commercialization of these AI algorithms, very few are currently approved for use in routine clinical practice.

Within cardiology, the future integration of AI seems inevitable, especially in the field of nuclear cardiology. Myocardial perfusion imaging (MPI) with SPECT or PET is a well-established non-invasive diagnostic and prognostic tools for the evaluation of patients with known or suspected coronary artery disease $(\mathrm{CAD})^{6,7}$. The study by Zheng et al. ${ }^{2}$ is not directed toward interpretation of MPI images. This program evaluated the efficacy of cNLP in abstracting data and key variables, which can be fed directly to a data base for statistical analysis from unstructured and unanalyzable free hand reports of MPI data. They analyzed the prognostic value of this abstracted data for predicting future adverse cardiac events in their study population and found it to be similar to previous studies, which used structured reporting or manually abstracted data. This helps to validate the accuracy of NLP abstracted data in retaining the prognostic value of the MPI. The current recommendations by the ASNC, IAC and other professional organizations is to generate structured reports of MPI studies, which brings a degree of uniformity and standardization in the use of terminology across various readers and institutions. Several institutions and readers continue to use free dictation to interpret nuclear imaging studies. This poses a significant challenge to use these data for research studies. This requires a rigorous, time consuming, and resource intense manual data abstracting. The authors of this manuscript have developed an automated program to abstract key elements of nuclear imaging data from the non-structured reports generated by free dictation by multiple image readers using NLP. The authors used data from approximately 17,000 nuclear stress test studies over a period of 4 years from patients who presented to the emergency department in Kaiser Permanente South California Hospitals with chest pain and underwent stress MPI within 30 days of discharge as a part of their clinical care. Nuclear stress tests were interpreted by readers using free dictation, without the use of structured reporting. This highlights the important role of AI in categorizing, extracting, and interpreting clinical information from large and complex unstructured data. Prediction of patient-specific future cardiovascular events is a difficult task given a large number of clinical, imaging, and electrocardiographic data available. While it is cumbersome, time consuming, and expensive to manually abstract data from a large number of unstructured reports, NLP can potentially accomplish this task with relative ease. However, it is important to recognize the narrow and limited scope of such algorithms. An NLP algorithm developed with conventional NLP platform for MPI studies would not be useful for abstracting data from coronary angiograms or mammograms. One would require separate algorithms for each testing modality. However, currently a new and very intriguing NLP platform called GPT-3 is under development. $^{7}$ This has a very massive platform, where extremely huge amounts of data (almost all extant literature) as well as programming algorithms can be fed. This platform can learn from the existing computer algorithms and automatically write new algorithms for new but similar situations. Perhaps, if several NLP algorithms for abstracting data from different kind of medical reports are fed into this platform, this might be able to automatically write a new algorithm for abstracting data from yet another kind of medical report. Although, very tantalizing, this platform is still in its infancy and requires massive amount of computing power, expertise, and unlimited financial resources.

Finally, it is worth noting that while AI aims to be precise in its clinical interpretation, it is not infallible. AI performs what it has learnt, and therefore, inaccuracies or mistakes in learning will lead to poor performance of the program. When GPT-3 was asked to write an article on a common topic, after it was fed the extant literature including published and unpublished books, magazine articles, blog postings, and facebook comments, it had multiple statements with obscenities, profanities, as well 
as bigotries. Well, after all the program learnt it from the information fed to it. It cannot differentiate between good and bad information. Ultimately, a human must oversee and take responsibility for the final result. NLP would not be able to correct the inaccuracies or judgment errors of the image readers.

Finally, the most important tool in a physician's repertoire is empathy, compassion, and an ability to build interpersonal doctor-patient relationship. AI is unlikely to replace these attributes of human touch that only a physician can provide. In the next decade, we will reach a paradigm shift in clinical medicine. An integration of $\mathrm{AI}$ in medicine, particularly in medical imaging is undisputed. Nevertheless, it remains for us to determine to which extent and how AI systems will be integrated into health care and clinical medicine. Most importantly, we must recognize that even the smartest algorithm will not be able to replace physicians at least in the foreseeable future, but will rather work quite effectively to complement the work of physicians.

\section{Disclosure}

None of the authors have any conflict of interest.

\section{References}

1. Artificial intelligence. Merriam-Webster.com Dictionary: MerriamWebster.

2. Zheng C, Sun BC, Wu YL, et al. Automated abstraction of myocardial perfusion imaging reports using natural language processing. J Nucl Cardiol. 2020. https://doi.org/10.1007/s12350020-02401-z.

3. Chang HY, Jung CK, Woo JI, et al. Artificial Intelligence in Pathology. J Pathol Transl Med. 2019;53(1):1-12. https://doi.org/ 10.4132/jptm.2018.12.16.

4. Hosny A, Parmar C, Quackenbush J, Schwartz LH, Aerts HJWL. Artificial intelligence in radiology. Nat Rev Cancer. 2018;18(8):500-10. https://doi.org/10.1038/s41568-018-0016-5.

5. Jin CY. A review of AI technologies for wearable devices. IOP Conf Ser Mater Sci Eng. 2019;688:44072. https://doi.org/10.1088/ 1757-899x/688/4/044072.

6. Bullock-Palmer R. The Evolution of the Role for Artificial Intelligence in Nuclear Cardiology. https://www.acc.org/latest-in-c ardiology/articles/2019/07/25/08/34/the-evolution-of-the-role-for-ar tificial-intelligence-in-nuclear-cardiology

7. Seetharam K, Shresthra S, Mills JD, Sengupta PP. Artificial intelligence in nuclear cardiology: adding value to prognostication. Curr Cardiovasc Imaging Rep. 2019;12:14.

Publisher's Note Springer Nature remains neutral with regard to jurisdictional claims in published maps and institutional affiliations. 